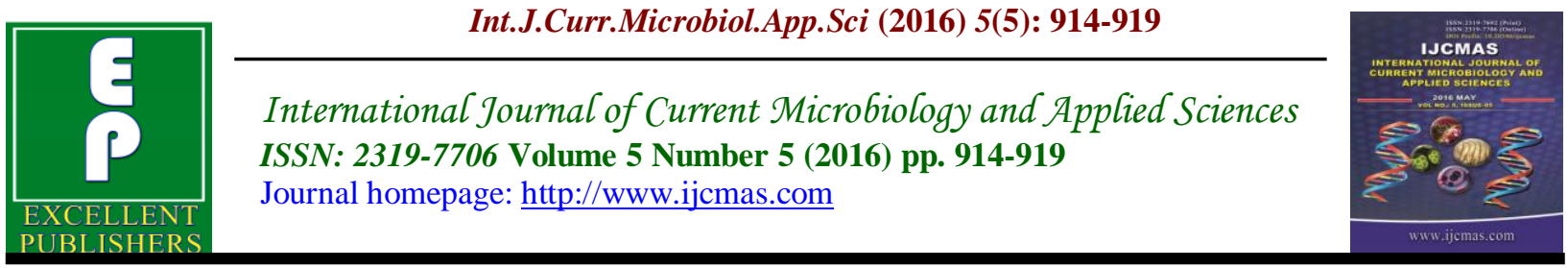

Original Research Article

http://dx.doi.org/10.20546/ijcmas.2016.505.095

\title{
Serological Follow Up for Confirmation of Current Leptospirosis
}

\author{
J. Padma Kumari*, K. Usha Krishnan and T. Sheila Doris \\ Government Medical College, Omandurar Government Estate, Chennai, India \\ Madras Medical College, Chennai, India \\ *Corresponding author
}

\begin{abstract}
A B S T R A C T
Keywords

Leptospirosis,

MSAT,

MAT,

paired sera,

follow up.

Article Info

Accepted:

22 April 2016

Available Online:

10 May 2016

Leptospirosis is becoming an increasingly significant public health problem, particularly in tropical developing countries. The clinical diagnosis of leptospirosis is difficult due to the varied presentation of the symptoms of the disease and thus laboratory methods are needed to confirm it. Serology becomes an important diagnostic tool from the moment antibodies appear in detectable quantities in blood. Microscopic Agglutination Test(MAT) is the gold standard serological test but a single positive titre is, not diagnostic due to asymptomatic, past infection and cross reacting antibodies to other illness and should ideally be performed on paired serum samples and a demonstration of four-fold rise in titre of antibodies is essential to confirm current illness. The aim of the present study is to confirm the current leptospiral infection by demonstrating four- fold rise in titre of antibodies using paired sera by Microscopic Agglutination Test. The study population were 1209 patients from Chennai, with fever of one week duration with signs and symptoms of Leptospirosis and were screened for Leptospirosis by Macroscopic Slide Agglutination Test(MSAT). Out of 1209 patients, 237 patients who were positive by screening test MSAT were followed-up to obtain paired serum sample. Second sample was collected from 158 patients. The acute phase serum sample and the paired sera were analysed by serological test MAT. Out of 158 cases followed up, 142(89\%) cases showed a four fold rise in titre of antibodies and were confirmed as having current leptospiral infection. If only a single sample was used to diagnose leptospirosis 16 cases would have been false positive for leptospirosis and the true cause of the febrile illness would have been missed. So it is mandatory that we examine two samples one in acute phase and one during convalescent phase thus confirming the current leptospiral infection.
\end{abstract}

\section{Introduction}

Leptospirosis is a globally widespread zoonotic disease. It is most commonly seen in tropical and subtropical countries. Human hosts commonly acquire infection through skin abrasions and mucosal surfaces following contact with water or soil contaminated with urine of infected rodents or other mammals. It presents with signs and symptoms similar to other diseases like influenza, dengue fever, hepatitis, meningitis and mimic them. Clinical diagnosis in tropical regions is difficult 
where similar acute illnesses are very commonly encountered.

Hence accurate diagnosis of leptospirosis is important for proper treatment especially in case of severe illness. Dark- field microscopy of blood interpretation needs technical experience as Brownian movement of red blood cell membranes and other artifacts can resemble motile living leptospires. The findings must be regarded as provisional and should be confirmed with the results of culture and serological tests. Isolation of Leptospira is difficult, it is time consuming and labour intensive. The organism requires a complex culture media and the generation time is also prolonged.

Serology becomes an important diagnostic tool from the moment antibodies appear in detectable quantities in blood. MSAT is a rapid serological test and is most useful as a screening test in acute phase of illness but needs to be confirmed by MAT.

MAT is the gold standard laboratory test in the diagnosis of leptospirosis due to its unsurpassed diagnostic specificity. In endemic areas, where previous mild or severe infections are common a single high titre in MAT would inevitably result in a false positive. (In some individuals MAT titres of 1:1600 have been known to persist for a few years).MAT is ideally performed on paired serum samples (acute and convalescent).Repeat samples (ideally demonstrating a four-fold rise in titre or seroconversion) are usually necessary to confirm the current illness.

The criterion for a definite diagnosis of current leptospiral infection is a four-fold rise in titre or seroconversion (Megan et al.,). This would help diagnose and confirmation of the infection and initiation of appropriate antibiotics which will reduce the morbidity of the affected patients and duration of their hospital stay. The present study will establish the role of paired sera in diagnosis and confirmation of leptospirosis and its role in differentiating leptospirosis from other acute febrile illnesses, especially in severe illness where appropriate antibiotic therapy is needed to prevent morbidity and mortality.

\section{Materials and Methods}

The study was conducted in Leptospirosis Research Cell, Madras Medical College and hospital over a period of one year duration from November 2012 to October 2013 after getting Ethical clearance. 1209 patients and who attended Rajiv Gandhi Government General Hospital, with fever within of one week duration, having signs and symptoms of leptospirosis were included in the study.

The demographic and clinical profile of the patients involved in the study was obtained by standard questionnaire. The serovars of leptospira used for MSAT and MAT are australis, autumnalis, canicola, grippotyphosa, hebdomadis, icterohaemorrhagiae, lousiana, bataviae, sejroe, pomona and semaranga which were locally prevalent serovars. The above standard strains were obtained from Royal Tropical Institute, Nederlands and subcultured in LRC, Institute of Microbiology, M.M.C. Under aseptic precautions $5 \mathrm{ml}$ of venous blood sample was collected from patients with 5-6 days of fever in a clean dry test tube and transported immediately to the laboratory. Blood was centrifuged, serum separated and was subjected to serological test MSAT.

MSAT was done using a dense suspension of killed Leptospires was mixed with a drop of serum on a slide and rotated on a rotator (120rpm) for 4 minutes. It was then 
examined by naked eye for presence of agglutination. Clumps of agglutination with complete clearing of leptospiral antigen was considered significant.

MSAT was positive among 237 patients. The positive samples were stored at $-20^{\circ} \mathrm{C}$ for further analysis by MAT. The 237 patients who were positive by screening test MSAT were followed-up to obtain paired serum sample. Second sample was collected from 158 patients. Due to various reasons from 79 patients the second sample was not obtained. The patients were followed-up for the second sample (paired sera) after one week of collecting the first sample.

Agglutination test done with live cultures of locally prevalent serovars of leptospires were used in MAT. The highest dilution of serum antigen mixture, which showed $50 \%$ agglutination, was taken as the end titre of the serum for that particular antigen. The cut-off point for a positive MAT was a titre of $\geq 1: 80$.

A serologically confirmed case of current leptospirosis is a four - fold rise in titre between the serum samples collected during the early days of illness and during convalescent phase run in parallel.

\section{Results and Discussion}

1209 patients were screened for Leptospirosis by serological test MSAT.237 patients were positive by MSAT. They were followed up for the second sample. Paired sera was obtained from 158 patients.

237 of the screened patients were positive for leptospirosis

Out of 158 cases followed up, 142(89\%) cases were confirmed as having current leptospiral infection i.e, out of 158 patients 142 patients showed a four fold rise in titre of leptospiral antibodies.

142 patients suffered from current leptospiral infection who were true positives and 16 of the cases were false positive cases detected by serological follow up by MAT.

Chennai is one of the most populous metropolitan city in India. Chennai has a long coastline and is the home of several millions of people. Due to rapid changes in the ecological system many zoonotic diseases have caused epidemics. Leptospirosis is one of them and is endemic in Chennai. The infection is of public health importance due to its morbidity and mortality.

Leptospirosis mimics many other diseases in its atypical presenting symptoms and clinical features and thus laboratory diagnosis of the disease is of utmost importance. Serological test are the test most commonly used to diagnose leptospirosis due to difficulty in its isolation, lack of sensitivity and specificity in dark field microscopy in laboratory diagnosis of leptospirosis (Vijayachari et al., 2001).

The present study was conducted over a period of one year duration from November 2012 to October 2013. Out of 1209 cases in Chennai city with clinical features suggestive of leptospirosis 237 cases were positive by MSAT is a rapid test used to identify if a person is infected or not and the test detects the $\operatorname{IgM}$ antibodies. Galton et al., in his study found that Patoc strain with killed antigen used for MSAT was more sensitive during the second week of illness. Usefulness of the test as a screening test was evaluated by Sumathi et al. 
Table.1 MSAT result among study population screened for leptospirosis $(n=1209)$

\begin{tabular}{|c|c|c|}
\hline Total samples & Positive & Negative \\
\hline 1209 & 237 & 972 \\
\hline
\end{tabular}

Table.2 Results of Paired Serum Samples by MAT (N=142)

\begin{tabular}{|c|c|c|c|}
\hline Dilutions & $\mathbf{1}^{\text {st }}$ Sample & $\begin{array}{c}2^{\text {nd }} \text { sample } \\
\text { (with four fold } \\
\text { rise) }\end{array}$ & $\begin{array}{c}2^{\text {nd }} \text { sample (without four } \\
\text { fold rise) }\end{array}$ \\
\hline Below 1 in 80 & 8 & 5 & 3 \\
\hline 1 in 80 & 108 & 98 & 10 \\
\hline 1 in 160 & 42 & 39 & 03 \\
\hline
\end{tabular}

Fig.1 Current Leptospiral Infection among Followed Up Patients

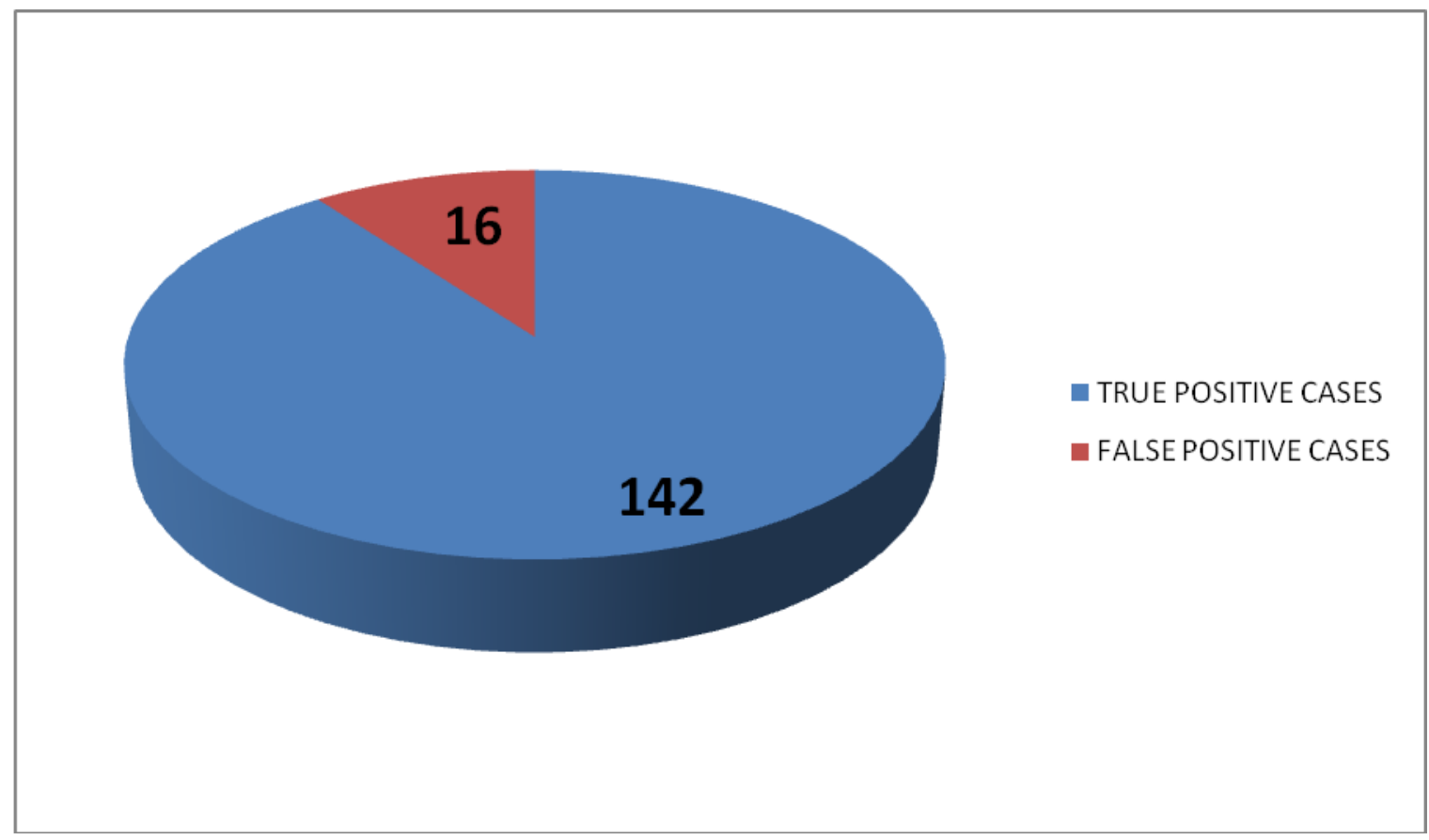

MSAT is the serological test that is easy to perform and read. The antigen is broadly reactive. It is stable for six month at $4{ }^{\circ} \mathrm{C}$ to $8^{\circ} \mathrm{C}$. It is positive in the early stage of the disease but a high percentage of false positive reactions are observed. The false negative reactions are comparatively low.

Out of 158 cases followed up 142(89.8\%) cases were confirmed of having current leptospiral infection by using paired serum sample collected at an interval of one week. If only a single sample was used to diagnose leptospirosis 16 cases would have been false positive for leptospirosis and the true cause of the febrile illness would have been missed. So it is mandatory that we examine two samples one in acute phase and one during convalescent phase thus confirming the diagnosis. 
The false positive cases may be due to residual antibodies that developed during past or asymptomatic infection or due to cross reacting antibodies to other febrile illness. Thus to avoid false positive results and to avoid unnecessary treatment it is mandatory that a paired serum samples (acute and convalescent) be tested for confirming the diagnosis of leptospirosis.

MAT is the gold standard serological test for confirming leptospirosis. The criterion for a definite diagnosis of current leptospiral infection is a four- fold rise in titre or seroconversion Because of the delay in antibody development (5 days after onset of first symptoms) single early blood samples may not have any detectable antibody and the negative result could be equally misleading (Shivakumar et al.,). The antibodies appear during the $5^{\text {th }}$ day of illness and so the serological tests may be negative when tested before that period or may test positive due to asymptomatic, mild leptospirosis or past leptospirosis or due to cross reacting antibodies of other febrile illness.

Therefore it is mandatory that a second serum sample(convalescent sera) be collected and tested in parallel with the $1^{\text {st }}$ sample (acute phase sera) and demonstrate a four- fold rise in titre thus confirming the diagnosis of current leptospirosis. Thus at least two serum samples taken at least 4 to 5 days apart should be examined before a negative result can be confirmed. The interval between paired samples may be short, provided they are taken very early in the acute phase and then a few days later, as in our patients.

However, this requires patients to have sought medical assistance within a few days of the onset of symptoms and a high degree of clinical suspicion on the part of the physician. MAT titres may take several years to decline. This of considerable value in epidemiological studies.

Although the MAT is the test for choice for serological surveys, it is complicated, has to be permanently established, and uses numerous serovars which must be maintained aseptically in stock culture. Normally it is used daily or weekly. Its main advantage is that it gives a good indication of infecting serogroup, especially late in illness or in surveys, since it detects serovar specific antibodies which tend to peak later.

\section{References}

Faine, S. 1982. Guidelines for the control of leptospirosis. World Health Organisation, Geneva, Switzerland.

Feign, R.D., Anderson, D.C., Health, C.W. 1975. Human leptospirosis. Crit. Rev. Clin. Lab. Sci., 5: 413-467.

Kee, S.H., Kim, I.S., Choi, M.S., Chang, W.H. 1994. Detection of leptospiral DNA by PCR. J. Clin. Microbiol., 32: 1035-9.

Leptospirosis Laboratory Manual, Regional Medical Research Centre: Port Blair, Andaman 2013.

Megan, E., Reller, Champika Bodinayake, Ajith Nagahawatte, Vasantha Devasiri, Wasantha KodikaraArachichi, John, J., Strouse, Judith, E., Flom, J., Stephen Dumler, Christopher, W., Woods. 2011. Leptospirosis as Frequent Cause of Acute Febrile Illness in Southern SriLanka. J. Clin. Pathol., Volume 9, Number 9, 29(3): 198-202.

Prescott Textbook of Medical Microbiology. $8^{\text {th }}$ edition pg 369-374.

Ratnam, S., Subramanian, S., Madanagopalan, N., Sundararaj, T., Jayanthi, V. 1983. Isolation of leptospires and demonstration of antibodies in human leptospirosis in 
Madras India. Trans. Royal Soc. Trop. Med. Hyg., 77: 455-458.

Romero, E.C., Caly, C.R., Yasuda, P.H. 1998. The persistence of leptospiral agglutinins titers in human sera diagnosed by the microscopic agglutination test. Rev. Inst. Med. Trop. Sao Paulo, 40: 183-184.

Shivakumar, S., Krishnakumar, B. 2006. Diagnosis of Leptospirosis- Role of MAT. J. Assoc. Phys. India, 4: 338339.

Sumathi, G., Chinari Pradeep, K.S.,
Shivakumar, S. 1997. MSAT-A Screening test for leptospirosis. Ind. J. Med. Microbiol., 15(2):84.

Sumathi, G,. Pradeep Kumar Subudhi, C.H., Helen, P.S., Manuel., Kalpana, Shivakumar, S., Suguna Rajendran, Muthusethupathi, M.A. 1995. Serodiagnosis of Leptospirosis - A Madras Study. Ind. J. Med. Microbiol., 13(4):192-195.

Vijayachari, P. 2007. Leptospirosis Laboratory manual, World Health Organization.

\section{How to cite this article:}

Padma Kumari, J., K. Usha Krishnan and Sheila Doris, T. 2016. Serological Follow Up for Confirmation of Current Leptospirosis. Int.J.Curr.Microbiol.App.Sci. 5(5): 914-919. doi: http://dx.doi.org/10.20546/ijcmas.2016.505.095 